International Journal of

BioScience and Applications

\title{
A case of radiculopathy in a young patient with spondylolysis without prominent nerve root compression
}

\author{
Cheol-Hyeong Lee ${ }^{1}$, Ki-Hyun Jang ${ }^{1}$ and Yeon-Dong Kim ${ }^{1,2}$ \\ ${ }^{1}$ Department of Anesthesiology and Pain Medicine, Wonkwang University School of Medicine, Wonkwang \\ University Hospital, Iksan, Korea \\ ${ }^{2}$ Jesaeng-Euise Clinical Anatomy Center, Wonkwang University School of Medicine, Iksan, Korea
}

\begin{abstract}
In general, in patients with radiating pain, nerve root compression is suspected, and the cause has been treated under the diagnosis of a herniated lumbar disc or spinal stenosis. However, inflammation around the nerve root alone can stimulate the nerve root by inflammatory cytokines, resulting in radiculopathy. In this case report, we report radiculopathy in a patient diagnosed with spondylolysis without disc degenerative change or protrusion. Also, the pathogenesis of low back pain and radiating pain is reviewed through literature.
\end{abstract}

\section{Index Terms}

Facet joint, Imaging test, Low back pain, Radiating pain, Radiculopathy, Spondylolysis, ,

Corresponding author : Yeon-Dong Kim

kydpain@wku.ac.kr

- Manuscript received January 15, 2021.

- Revised February 10, 2021 ; Accepted March 1, 2021.

- Date of publication March 30, 2021.

(c) The Academic Society of Convergence Science Inc.

2619-8363 $\odot 2021$ IJBSA. Personal use is permitted, but republication/redistribution requires IJBSA permission. 


\section{INTRODUCTION}

Low back pain combined with radicular symptoms remains one of the most common but challenging musculoskeletal problems for its therapeutic management in clinical practice. As there are various medical conditions that cause low back pain or lower extremity pain, the differential diagnosis for low back pain is broad and should include, among other diagnoses, lumbosacral radiculopathy [1]. In patients complaining of leg pain greater than low back pain, lumbar radiculopathy or foraminal stenosis is described as the most common etiology [2].

Traditionally, lumbosacral radiculopathy is the term used to represent a pain syndrome caused by mechanical compression or irritation of the spinal nerve root. The most cases, It is caused by direct neural compression of nerve roots from herniated lumbar disc, narrowing of the spinal foramen or central canal, and degenerative change of the spinal vertebrae [3].

The facet joints, which are synovial and plane joints between the articular processes of two vertically adjacent vertebrae, can cause lumbar back pain. The most common cause of facet joint disease is a degenerative change of the spine, also known as spondylosis. 'Spondylosis' is sometimes used interchangeably with spondylitis, hypertrophic arthritis, or arthrosis, and 'Spondylolysis' refers to a defect of pars interarticularis [4]. The main clinical symptom of lumbar spondylolysis is low back pain, and radiculopathy rarely occurs in the absence of nerve root compression, especially in young patients [5]. However, recent studies have raised the possibility that radiculopathy may occur only with a problem of the facet joint without compression of the mechanical nerve root.

Here is a case report with a review of the literature, in which to report a case of prominent radiculopathy and its treatment in a patient showing only spondylolysis on imaging tests.

\section{CASE}

A 21-year-old male patient was referred to the pain clinic center for pain in his low back and right lower extremities. He was of the average physique with $178 \mathrm{~cm}$ height and $78 \mathrm{~kg}$ weight. The patient's medical history did not show any particularities, and his occupation was a college student. Prior to the onset of symptoms, he enjoyed light exercise in his usual routine but did not overuse his body.

Symptoms started 4 months ago without any suspicious medical event, and the patient complained of low back pain and stiffness of the lumbar spine.
He also complained of a tingling sensation on the right posterolateral thigh and severe radiating pain extending to the right foot dorsum. Also, the patient complained of occasional lancinating pain and numbness of the lateral thigh.

The patient testified that he was diagnosed with suspected lumbar disc herniation based on his symptoms and was treated at a local orthopedic and neurosurgeon. He was prescribed medication from the clinics that he was treated, but it did not work, and he appealed that his symptoms had worsened recently. At the time of the visit, he was taking Celecoxib $200 \mathrm{mg}$, Pregabalin $75 \mathrm{mg}$, Eperisone 50 $\mathrm{mg}$, and Rebamipide $100 \mathrm{mg}$ BID. And he also had received a lumbar epidural block twice and a selective nerve root block once over the past 3 months for symptom relief, but the effect was limited.

On physical examination, he complained of mild tenderness in the right lumbar region, and pain was induced at 30 degrees SLRT. He also showed limp walking due to worsening pain when walking. The most intense pain was reported to have reached an NRS pain score of $8 / 10$.

Initially, he underwent a lumbar x-ray for diagnosis. The radiographic image revealed defects of both pars interarticularis on the L5 level (figure 1A, 1B). At this point, the patient could be diagnosed with spondylolysis, but it was difficult to explain the severe radiating pain from this $\mathrm{x}$-ray image alone. Therefore, a lumbar MRI was performed with the consent of the patient.

In the sagittal view, the T2 MRI image showed high signal intensity in the pars interarticularis region and low signal intensity in T1(Figure 2A, 2B). This finding was thought to suggest an inflammatory or degenerative bone marrow change around the patient's facet joint. No degenerative change or protrusion of intervertebral disc was observed at any level(Figure 2C). In the axial view, right side spondylotic changes are observed. (figure 3A, 3B)

After confirming the MRI image, a diagnostic facet joint block was decided to determine whether the patient's symptoms were caused by the facet joint. Facet joint block was applied at L4/5 and L5/S1 levels. It was performed with a $25-\mathrm{G}$ needle, $90 \mathrm{~mm}$ long, and $5 \mathrm{mg}$ of dexamethasone and $0.5 \mathrm{ml}$ of $0.5 \%$ mepivacaine were injected(figure 4A, 4B). The entire procedures were performed under fluoroscopy, and No adverse events were observed.

At the one-week follow-up after facet joint block, the patient reported that the pain was improved by more than $50 \%$, and the radiating pain during walking almost disappeared. In order to improve the remaining symptoms, the same facet joint block was performed once again, and physical therapy and 
exercise were recommended to the patient. At follow-up after 1 month, the patient's symptoms improved, and there was only mild discomfort with low back once or twice a day, and he said that there was no problem with his daily life. After 3 months, confirming that the patient's pain had completely disappeared, the treatment was terminated.
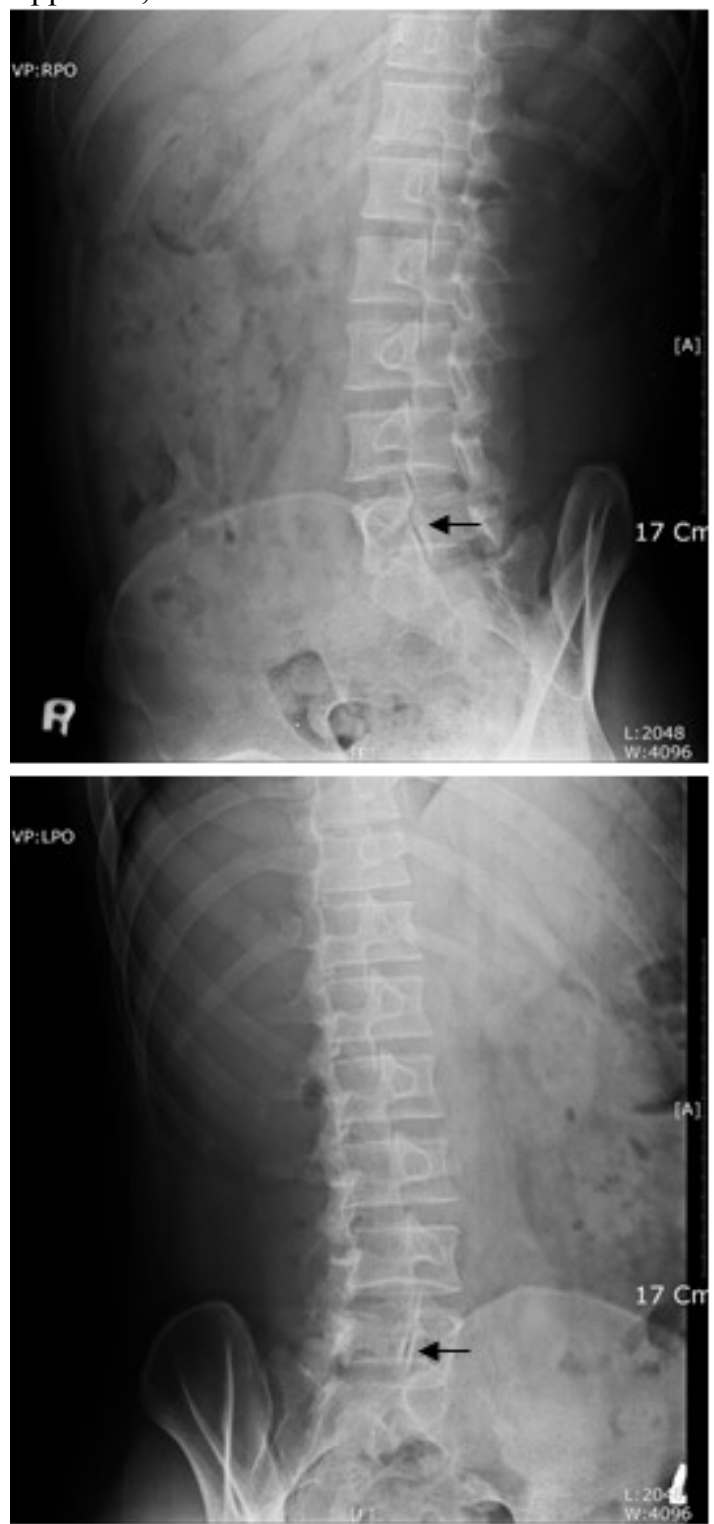

Fig. 1. The X-ray image revealed defects of both pars interarticularis on the L5 level (Black arrow).
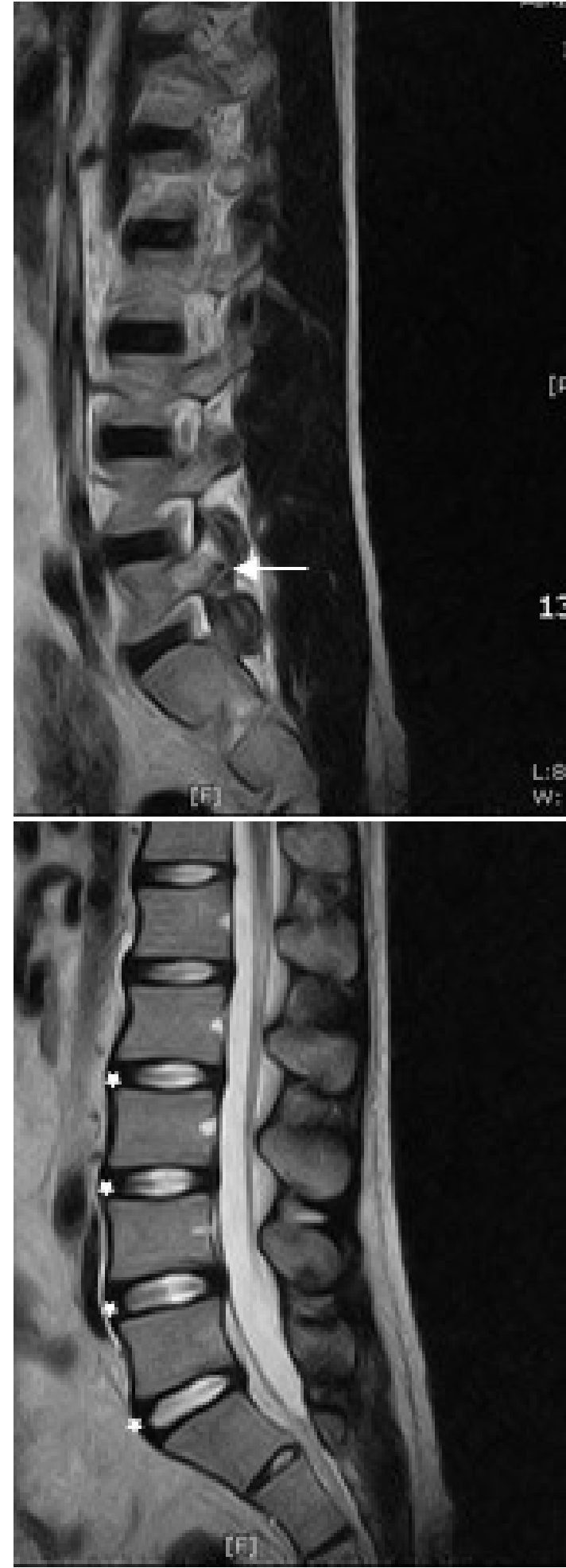

Fig. 2. Sagittal view of the lumbar MRI image. Signal changes in the pars interarticularis (white arrows). Normal intervertebral discs (white stars). 

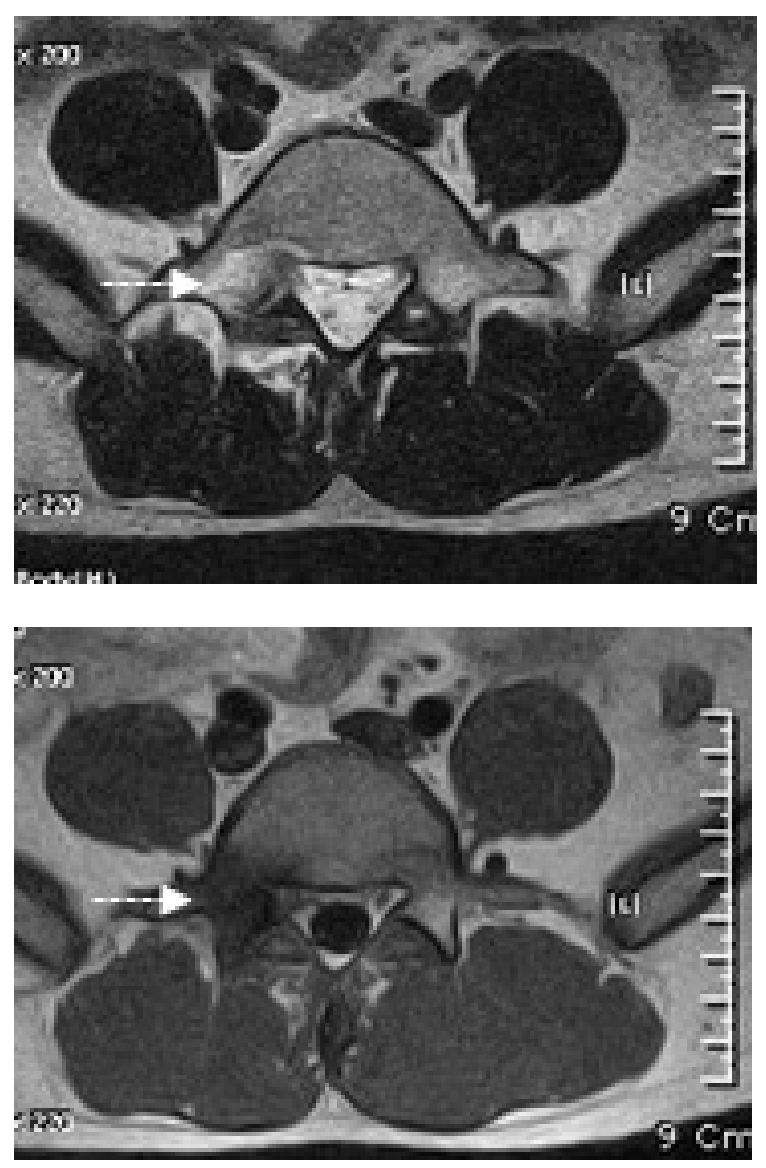

Fig. 3. Axial view of the lumbar MRI image. Spondylotic changes on the right side of spine (Dotted arrows).

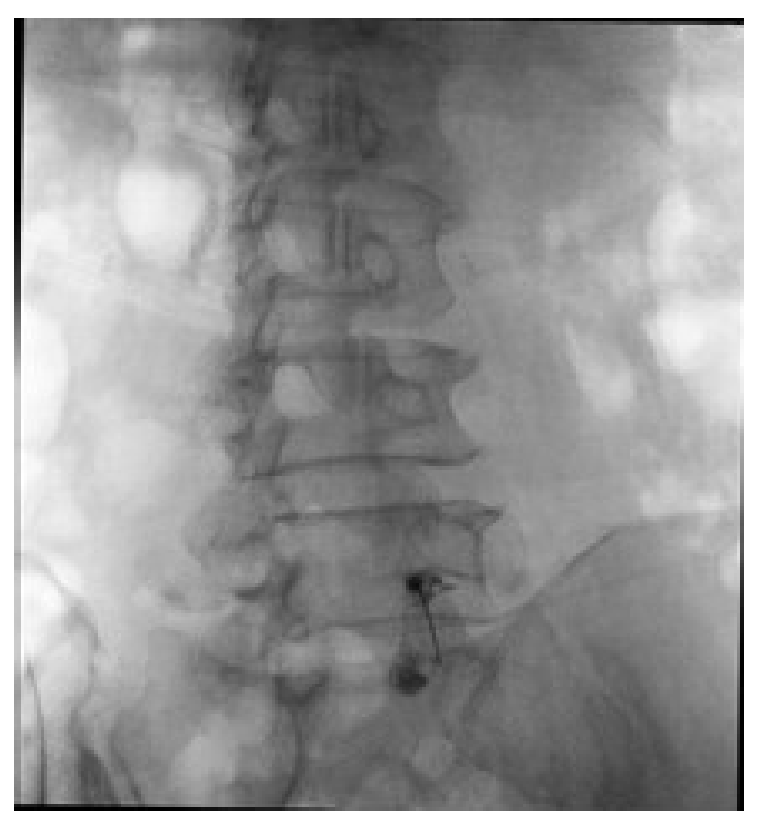

Fig. 4. Fluoroscopic image of facet joint block performed to the patient.

\section{DISCUSSION}

Although there are differences between studies, low back pain is common enough that $80-85 \%$ of people experience it at least once in their lifetime, but it is difficult to diagnose the exact cause. [6] There is no agreement on various definitions of low back pain, and the anatomical complexity of the spine and the diversity of clinical manifestations of spine-related diseases are factors that make diagnosis difficult [7]. Therefore, classifying low back pain into radiating back pain and non-radiating back pain helps the general practitioner plan a treatment strategy [8]. In general, radiating low back pain is caused by radiculopathy, and the prognosis is worse in terms of pain intensity, quality of life, and disability [9]. Therefore, it will be of great help in improving the prognosis of patients or reducing medical costs to distinguish which spinal diseases cause radiating pain and which diseases do not.

The cause of radiculopathy is mechanically compression or irritation of the nerve root. Since spondylolysis itself means only a defect in the pars interarticularis, it does not compress the nerve root so that it may cause low back pain, but it may not be the cause of radiculopathy. With increasing age, progression of spondylolysis may lead to spine instability, spondylolisthesis, or overgrown bony spurs, results in compression of the nerve root. However, this is not common in the early stages of degeneration $[10,11]$.

Nevertheless, patients who show radiculopathy without clear nerve root compression on imaging, such as the patient in this case, can often be encountered in clinical settings. In addition, in some patients with lumbar spondylolysis, clearly confirmed nerve root compression by imaging tests remains asymptomatic, and even though nerve root compression has not been relieved, radiating symptoms can be resolved with only nonoperative treatment. These patients suggest that mechanical compression is not a non-only factor in radiculopathy [12]. These cases can be explained by the effect of chemical stimulation of the nerve root rather than mechanical compression. Several studies have already reported that not only mechanical compression but also inflammatory cytokines caused by intervertebral disc herniation are involved in radiculopathy $[13,14]$. In these studies, the correlation between chemical factors and radiculopathy was limited to disc disease, but in this case, radiculopathy occurred in a patient with spondylolysis that progressed without degenerative change of the intervertebral disc.

This case suggests that inflammatory changes in the facet joint or surrounding tissues may also cause 
radiculopathy. The facet joint is a synovial joint like any other joint in the extremities. It means that each facet joints are surrounded by a capsule of connective tissue filled with fluid. Therefore, In the case of spondylotic or osteoarthritic changes of the facet joint, the inflamed synovium in a facet joint can be a source of chemical factors for radiculopathy. A study by Igarashi et al. also suggested a significant correlation between the level of inflammatory cytokines in the facet joint synovium and the degree of leg symptoms [15].

Conversely, it suggests that the degeneration of the facet joint without inflammation is not related to the symptoms of the legs, which corresponds to the clinical fact that some patients remain asymptomatic despite the degeneration of the facet joint in the diagnostic image [16]. That is, although many physicians rely on radiology tests, Imaging findings are weakly related to symptoms.

Therefore, diagnosing and treating low back pain and radicular pain presents many difficulties and still remains challenging for physicians. In particular, the lumbar facet joint problem is difficult to diagnose radiologically because there are no pathological findings to be found [17].

Nevertheless, because plain $\mathrm{x}$-rays are insensitive to the pathology of the spine, magnetic resonance imaging (MRI) is increasingly used for low back pain. Still, most of the utilization is outside the appropriateness guidelines [18]. According to the recommendations of the American College of Radiology, MRI should not be performed for LBP imaging within the first 6 weeks unless there are unclear clinical factors or neurological defects.

However, as in the patient's situation in this case, if the radiculopathy symptoms do not respond to conservative treatment even after more than 6 weeks of symptom onset, it is worth taking MRI aggressively. Because MRI is helpful in confirming compression and highly sensitive to confirming inflammatory changes in surrounding tissues even in the absence of mechanical compression [19].

In this case, MRI was performed 4 months after the onset of symptoms, and during that time, the patient was diagnosed and treated only with a herniated lumbar disc. However, no degenerative changes were found in the intervertebral disc on MRI, and only spondylolysis was diagnosed. These points suggest that although history and clinical examination are still considered fundamental steps in diagnosing low back pain, appropriate neuroimaging studies should be accompanied.

\section{Conclusion}

Radiculopathy may also develop in patients with spondylolysis. This is due to inflammation around the affected nerve rather than compression of the nerve. While it is reasonable and common to make an initial impression based on the patient's clinical symptoms and treat accordingly, if the patient does not respond to treatment, reevaluating the patient with all possibilities in mind is a better way to treat the patient.

\section{REFERENCES}

[1] Alexander, C.E. and M. Varacallo, Lumbosacral radiculopathy. StatPearls [Internet], 2021.

[2] Della-Giustina, D.A., Emergency department evaluation and treatment of back pain. Emergency medicine clinics of North America, 1999. 17(4): p. 877-893.

[3] Tarulli, A.W. and E.M. Raynor, Lumbosacral radiculopathy. Neurologic clinics, 2007. 25(2): p. 387-405.

[4] Middleton, K. and D.E. Fish, Lumbar spondylosis: clinical presentation and treatment approaches. Current Reviews in Musculoskeletal Medicine, 2009. 2(2): p. 94-104.

[5] Sairyo, K., et al., Causes of radiculopathy in young athletes with spondylolysis. The American journal of sports medicine, 2010. 38(2): p. 357-362.

[6] Rubin, D.I., Epidemiology and risk factors for spine pain. Neurologic clinics, 2007. 25(2): p. 353-371.

[7] Fatoye, F., T. Gebrye, and I. Odeyemi, Real-world incidence and prevalence of low back pain using routinely collected data. Rheumatology international, 2019. 39(4): p. 619-626.

[8] Spijker-Huiges, A., et al., Radiating low back pain in general practice: incidence, prevalence, diagnosis, and long-term clinical course of illness. Scandinavian journal of primary health care, 2015. 33(1): p. 27-32.

[9] Konstantinou, K., et al., The impact of low backrelated leg pain on outcomes as compared with low back pain alone: a systematic review of the literature. The Clinical journal of pain, 2013. 29(7): p. 644-654.

[10] Gunzburg, R. and M. Szpalski, Spondylolysis, spondylolisthesis, and degenerative spondylolisthesis. 2006: Lippincott Williams \& Wilkins.

[11] Kushchayev, S.V., et al., ABCs of the degenerative spine. Insights into imaging, 2018. 9(2): p. 253-274.

[12] Ng, L., N. Chaudhary, and P. Sell, The efficacy of corticosteroids in periradicular infiltration for chronic radicular pain: a randomized, double-blind, controlled trial. Spine, 2005. 30(8): p. 857-862.

[13] Aoki, Y., et al., Local application of disc-related cytokines on spinal nerve roots. Spine, 2002. 27(15): p. 1614-1617.

[14] Hou, S.-X., et al., Chronic inflammation and compression of the dorsal root contribute to sciatica induced by the intervertebral disc herniation in rats. Pain, 2003. 105(1-2): p. 255-264.

[15] Igarashi, A., et al., Inflammatory cytokines released from the facet joint tissue in degenerative lumbar spinal disorders. Spine, 2004. 29(19): p. 2091-2095.

[16] Tachihara, H., et al., Does facet joint inflammation 
[17] induce radiculopathy?: an investigation using a rat model of lumbar facet joint inflammation. Spine, 2007. 32(4): p. 406-412.

[18] Jackson, R.P., R.R. Jacobs, and P.X. Montesano, 1988 Volvo award in clinical sciences. Facet joint injection in low-back pain. A prospective statistical study. Spine, 1988. 13(9): p. 966-971.

[19] Swedlow, A., et al., Increased costs and rates of use in the California workers' compensation system as a result of self-referral by physicians. New England Journal of Medicine, 1992. 327(21): p. 1502-1506.

[20] Rao, D., et al., The use of imaging in management of patients with low back pain. Journal of clinical imaging science, 2018. 8. 